\title{
Genre et commerce équitable au Cameroun*
}

\author{
Gérard Tchouassi**
}

\section{Résumé}

L'objet de cet article est d'analyser la littérature existante sur le genre pour montrer comment les principes de base du commerce équitable peuvent favoriser la révision de la place des femmes dans les transactions internationales ou leur accès au commerce mondial. À cet égard, à partir de l'hypothèse selon laquelle les femmes sont en majorité des créatrices d'entreprises du commerce équitable, nous avons analysé dans cet article les données sur le Cameroun en utilisant une approche socioéconomique. Nous sommes arrivé à la conclusion selon laquelle pour participer au commerce équitable un certain nombre de principes incontournables doivent être respectés dans but d'assurer l'égalité de genre. Le circuit de distribution permet d'établir le « juste prix » du produit éthique fabriqué qui tient compte des coûts réels de production et non de subir le prix des multinationales et des grandes chaînes de distribution.

\begin{abstract}
The article seeks, through an analysis of literature on gender, to illustrate how basic principles of fair trade can permit the reading of the position of women in international exchange or their access to world trade. Using the hypothesis that women are the main creators of fair trade enterprises in Cameroon, the paper employs a socio-economic approach to analyse relevant data on Cameroon. In
\end{abstract}

* L'auteur remercie les lauréats de l'Institut du CODESRIA sur le Genre-Session 2002 dont le thème était : «Globalisation, Genre et Commerce » et les évaluateurs anonymes de la revue pour leurs remarques pertinentes qui ont permis d'améliorer la qualité de cet article. Il reste seul responsable des erreurs et omissions qui subsisteraient.

** Docteur d'État en Sciences Economiques, Faculté des Sciences Economiques et de Gestion, Université de Yaoundé II, Cameroun. 
conclusion, the paper highlights certain basic principles to be respected in order to secure gender equality in fair trade. The distribution channel permits the setting up of a 'Fair Price' for goods produced that take into account ethics and the real costs of production, rather than simply coming under the influence of multinationals and big distributors present in the various sectors of production.

\section{Introduction}

Le commerce étant une activité d'échange de biens et de services entre des personnes physiques ou morales reste indispensable à la cohésion de toute société. L’organisation actuelle du commerce mondial se fait à l'insu du producteur et du consommateur : le producteur ne connaît pas la destination de son produit ; le consommateur ignore la provenance réelle des biens qu’il consomme. Au détriment du producteur et du consommateur, les intermédiaires les plus puissants (grandes marques commanditaires, grands groupes industriels, organismes financiers, grands distributeurs, centrales d'achat) imposent leurs règles, leurs méthodes de production, leurs prix, voire leurs produits aux producteurs comme aux consommateurs. Un nouveau modèle de commerce international socialement responsable attire l'attention et suscite des discussions optimistes au sein d'organisations communautaires, d'organisations non gouvernementales (ONG), de gouvernements, de collectivités et même d'organisations internationales. Comme alternative au modèle commercial dominant, le commerce équitable met en avant le bienêtre des producteurs du Sud, plutôt que les profits des intermédiaires, au centre des transactions commerciales internationales (Tadros et Malo 2002).

Comme un moyen de mettre en relation les hommes, les entreprises, les multinationales, les organismes, les institutions, etc., le commerce est devenu un enjeu de pouvoir et de profit privé lié à la spéculation à court terme. Inéquitable, cette forme du commerce met en exergue la relation «dominant/dominé ». Ce n’est pas le commerce en tant que tel qui fait problème, mais son utilisation comme outil de domination économique. Cette situation entraîne le déplacement des marges vers l'aval. En effet, les producteurs voient leur marge s'éroder sous la pression des industriels et des distributeurs, appauvrissant et excluant ainsi les producteurs au plan économique, dans l'organisation de leur travail et dans la satisfaction de leurs besoins essentiels, ainsi que ceux de leur famille. Ce commerce est responsable de la dégradation des termes de l'échange. Pendant que le prix des matières premières décroît systématiquement sur le marché mondial, le prix des produits finis, que les pays producteurs de ces matières premières importent des pays industrialisés, augmente.

Placés dans ce contexte, les producteurs produisent, transforment et fabriquent des biens dans des conditions de travail souvent inhumaines, voire 
d'esclavage. Les conséquences sont alors à déplorer tant pour eux que pour l'environnement économique, social, écologique et culturel. Cette réalité se vérifie dans l'ensemble du commerce, tant au niveau local, régional qu'international. Face à ce constat, l'objectif de l'entrepreneuriat social et solidaire ${ }^{1}$ des femmes, à travers le commerce équitable (Fair Trade) pour un développement durable, est de permettre aux producteurs et aux consommateurs de vivre durablement leur dignité, leur autonomie en restant indépendant et en retrouvant la maîtrise, c’est-à-dire le sens d'organisation de leurs activités.

Alors que les débats sur la mondialisation (Touna Mama 1998) occupent de plus en plus de place dans l'actualité, les petits producteurs ${ }^{2}$ et artisans du Sud restent soumis à une pression sauvage des lois du commerce. Les matières premières du sol et du sous-sol, qui font vivre une grande partie des salariés et producteurs du Sud, voient leur cours, sur le marché mondial, s’affaiblir d’année en année. Les marchés alimentaires sont très instables (fortes fluctuations des cours) et oligopolistiques (4 multinationales contrôlent 90 pour cent du marché du café). Par exemple le cours du café a atteint 0,55 euro la livre en octobre 2001 alors qu'il était de 1,69 euro en mars 1998. Pour les producteurs la pression des intermédiaires (les multinationales, les commanditaires, les grands groupes industriels, les grands distributeurs, etc.) est de plus en plus forte : les prix, les conditions de production, de travail et d'achat leurs sont imposés. Cette pression est d'autant plus écrasante pour les petits producteurs qui n'ont pas d'accès direct au marché mondial.

Dans ces conditions, un petit agriculteur ou artisan isolé du Sud peut-il vivre dignement et durablement de son travail ? Il est couramment obligé de travailler dans des conditions comparables à l'esclavage, faire travailler ses enfants, et renier son environnement social, économique, écologique et culturel. C'est pour barrer la voie à toutes ces formes de pressions que les mouvements « anti-mondialistes » et « altermondialistes » se sont constitués en associations et en entreprises sociales et solidaires pour défendre les producteurs et les consommateurs et ont impulsé le commerce équitable des produits éthiques en vue d'un développement durable. Des processus (par exemple la production des biens et des services) et des institutions (organisations, associations, communautés, collectivités, etc.) durables supposent que certains critères d'équité, d'éthique, d'égalité de sexe et de genre doivent être remplis. Ils ne doivent pas épuiser les ressources des générations futures. Les capacités des populations au sein des institutions doivent être renforcées en permanence (formation, apprentissage de techniques, etc.). Les responsabilités et les bénéfices doivent être partagés équitablement au sein des familles entre hommes et femmes, dans les communautés, dans les associations, etc. 
Peut-on alors considérer le commerce équitable comme un moyen pour les femmes de participer au commerce mondial, à l'échange mondial ? Dans cette contribution, on partira de la littérature existante sur le genre pour montrer comment les principes et les fondements du commerce équitable peuvent favoriser la révision de la place des femmes dans les transactions internationales ou leur accès au commerce mondial. En posant l'hypothèse selon laquelle les femmes sont en majorité des créatrices d'entreprises du commerce équitable, nous analysons les données de terrain en utilisant une approche socioéconomique. Nous traiterons, dans une première partie, de l'évolution des différentes approches sur le genre et dans une seconde, des fondements et des principes du commerce équitable en mettant en exergue la façon dont les femmes doivent accéder à l'échange international.

\section{Analyse conceptuelle du genre}

Dans les pays du Nord comme dans ceux du Sud, il ne peut y avoir commerce équitable pour un développement durable ${ }^{3}$ sans l'implication des femmes ou sans la prise en compte de leur rôle, de leur place, de leur participation et de leur contribution dans les échanges commerciaux, dans le processus de création de la valeur ajoutée et de la richesse. Pour cela, des approches ont été développées ici et là par des chercheurs féministes, des organisations internationales, etc. Du sexe biologique au genre, du concept d' « intégration des femmes au développement " (IFD) au concept de "genre et développement » (GED) en passant par celui de " femmes et développement » (FED), on examinera les conceptions et les approches assez récentes de l'évolution des théories sur le genre 4 .

Depuis le début du XXe siècle, la réflexion sur la relation entre le sexe et le genre a connu des moments importants, dont chacun correspond à la prééminence d'un modèle théorique particulier. Partis d'un modèle « unidimensionnel » enraciné dans la dimension biologique, les chercheurs ont élaboré des modèles « bi » ou " pluridimensionnels » pour rendre compte de la complexité croissante de la catégorie du genre et de ses composantes 5 .

Dans le modèle « unidimensionnel » qui s'est imposé avec constance jusqu'au milieu du siècle dernier, l'homme et la femme, le masculin et le féminin sont des pôles opposés d'un continuum. Tout ce qui se situe entre eux ne relève pas vraiment de la réalité humaine. Ce modèle s'appuie d'abord sur certaines notions relevant de l' « essentialisme biologique » (selon lequel l'homme et la femme ont une nature différente), ensuite sur une articulation « bipolaire » des genres en ordonnant une séparation des univers, des rôles et des identités respectifs des deux sexes et en prévoyant de sévères sanctions sociales pour l'individu qui échoue à se comporter en homme ou en 
femme « authentique ». Enfin, ce modèle est basé sur la cohésion des divers éléments constitutifs du genre (traits de personnalité, attitudes, systèmes de valeurs, schémas comportementaux de prédilection, etc.).

Sur les plans individuel et social, l'on a considéré qu'il existe une correspondance stricte entre l'individu et ce qu'il est censé être en vertu de son sexe biologique. En effet, tout homme serait masculin et naturellement enclin à s'investir dans les activités viriles trouvant leur expression la plus parfaite dans la politique, la guerre ou les activités relevant du domaine public. Toute femme serait féminine et spontanément prompte, ainsi que le confirme sa capacité à enfanter, à privilégier les activités domestiques (ménages) et privées (activités génératrices de revenus). Le cadre de référence est ici entièrement déterminé par le sexe biologique. Car tout écart comportemental ou psychologique par rapport à la norme définit l'identité des sexes tout en réglant leurs attentes réciproques. Ce qui apparaît tout simplement comme une transgression des lois naturelles. Ce modèle « unidimensionnel », dont on pourrait faire remonter l'origine à l'âge de la pierre et au-delà, pèse toujours lourdement sur le fonctionnement social et économique des pays industrialisés ${ }^{6}$ qui se comptent parmi les plus égalitaires.

Les premières théories « bidimensionnelles » apparurent dans les milieux universitaires au début des années 1970, dans le sillage du mouvement de libération, et adoptèrent après le terme de " genre » pour signifier que la masculinité et la féminité constituent des réalités indépendantes du sexe biologique. En effet, la politique de développement de l'époque était dominée, pour ce qui concerne les femmes, par l'approche dite de la "protection sociale », associée au modèle de la croissance accélérée. En effet, dans cette optique, le rôle de la femme était étroitement lié à la reproduction, c'est-àdire au devoir de la mère ou de l'épouse. On ne s'intéressait pas du tout à son travail en tant que productrice de la valeur ajoutée ou d'une activité génératrice de revenus. Une première évolution profonde a eu lieu pendant cette décennie sous l'effet combiné du mouvement féministe libéral aux États-Unis et d'un intérêt accru pour la question féminine dans les pays en développement, surtout avec la contribution de Ester Boserup et en particulier avec la création de la première Décennie des Nations Unies pour la Femme.

L’IFD a été développée dans les années 1970 et 1980 par les économistes comme Ester Boserup (1983). Cette approche met en exergue le fait que la contribution des femmes au développement a été méconnue et mal évaluée. En outre, elle préconise de rendre le développement plus efficace, plus pratique et plus réel en faisant participer directement ces actrices à son processus. L'approche intégrée de l'égalité entre les femmes et les hommes est une stratégie qui vise à obtenir l'égalité entre les sexes. Elle évalue les différents 
effets que toute action planifiée produit sur les femmes et les hommes et intègre les préoccupations et les expériences des uns et des autres à la conception, à la mise en œuvre, au contrôle et à l'évaluation des procédures et des programmes. Tout cela pour que les femmes et les hommes puissent bénéficier de manière égale des expériences et des programmes. Ainsi l'inégalité ne serait pas perturbée. En réalité, l’intégration n’exclut pas les activités spécifiques des femmes et les actions positives (BIT 2000).

La conception FED ou RFD (rôles des femmes dans le développement), prônée au début de la décennie 1980, se fonde sur l'argument selon lequel, les femmes participent aux mécanismes de développement, mais sur des bases mal définies et inégales. Il apparaît directement une marginalisation des femmes, qui est sans aucun doute très préjudiciable à un développement harmonieux efficace, équitable et durable. On envisageait déjà à ce moment d'améliorer leur accès aux ressources et leur implication au processus d'élaboration et de prise de décisions. Les nouvelles politiques qui procèdent de cette approche ont joué un rôle primordial jusqu’au milieu de la décennie et se poursuivent jusqu'à nos jours, avec comme priorités successives l'équité, la lutte contre la pauvreté et l'exclusion, l'efficacité et l'accès à l'autonomie. Pour les tenants de cette approche, c'est l'incapacité à prendre en compte le rôle producteur de la femme qui donne lieu à une utilisation inefficace des ressources. Le recours à la notion d'efficacité a permis de rompre avec les politiques de protection sociale menées avant l'application de la dite approche. Mais il a aussi été commandé par la nécessité de « vendre » le projet FED en matière d'égalité fondamentale aux agences de développement et aux organismes gouvernementaux.

L’optique GED, apparue dans les années 1980 et avec comme objectif la prise en compte de la notion d'égalité dans la conception et l'analyse de la question du développement durable, a permis de réparer les omissions et les discriminations à l'égard des femmes. Cette nouvelle approche se propose d’intégrer la dimension du genre dans la politique du développement pour en accroître l'efficacité et retrouver de nouvelles possibilités d'améliorer la distribution des ressources et d'accroître l'équité. L’innovation importante rattachée à cette vision tient au fait que les « femmes s’inquiètent de voir les problèmes féminins perçus sur le plan du sexe, différences biologiques par rapport aux hommes « et non sur le plan du genre » rôles sociaux, relations entre hommes et femmes et les forces qui perpétuent ces relations tout en les modifiant » (Tchamanbe 1999). Donc, la nouvelle approche privilégie l'analyse des relations entre ces deux acteurs sociaux, ce qui permet de remédier à certains défauts imputés au concept FED, notamment un manque de clairvoyance en matière de relations de pouvoir. Dans le contexte de l'ajustement 
structurel, des tentatives prometteuses et fondées sur l'efficacité ont été faites dans le cadre du GED pour transposer l'analyse selon les critères de sexe du plan de projets à celui de l'élaboration des politiques macro-économiques. A cet effet, le genre est souvent soumis à certains éléments de lecture néo-classiques comme les distorsions du marché, les rigidités, l'asymétrie de l'information', etc.

Alors que dans les deux premières approches, les femmes sont systématiquement subordonnées et confinées à des rôles secondaires, inférieurs à ceux des hommes, et que leurs besoins ont été satisfaits sans tenir compte du contexte général, l'approche GED permet de mettre en exergue, en guise de stratégie, la réduction des écarts en contribuant à l'amélioration de l'égalité et de l'équité entre les genres. En outre, l'approche reconnaît que les femmes font partie intégrante aujourd'hui de toutes les stratégies de développement. Seulement, tant que certains problèmes conceptuels et méthodologiques ne seront pas résolus, elle risque de ne pas atteindre son objectif initial d'intégration de l'égalité et de se retrouver dans le créneau institutionnel marginalisé officiellement libéré par le FED. L’enjeu n'est rien de moins que la viabilité opérationnelle et la crédibilité du concept même de GED. Toutefois, les questions de la prise en compte du genre et de l'intégration de la femme au processus de développement retiennent l'attention de certaines organisations internationales de développement. Ainsi et depuis quelques années, le Programme des Nations Unies pour le Développement (PNUD) a adopté deux nouveaux indicateurs synthétiques des disparités entre les deux sexes. Ce sont l'« indicateur sexospécifique du développement humain » (ISDH) et l' « indicateur de la participation des femmes » (IPF).

L' « indicateur de développement humain » (IDH) est la moyenne de trois indicateurs : la durée de vie, le niveau d'éducation et le produit intérieur brut (PIB) réel corrigé par habitant. L'ISDH se base sur ces critères en distinguant les deux sexes (masculin et féminin). L'indicateur de la durée de vie (IDV) est ainsi corrigé pour tenir compte de l'avantage biologique des femmes dans ce domaine. Calculer la part du PIB des hommes et des femmes semble plus complexe. En effet, le calcul se base sur leurs parts respectives dans la production des revenus du travail. Ces parts sont exprimées à partir du rapport entre la moyenne des salaires féminins et celle des salaires masculins, et à partir du pourcentage des femmes et des hommes au sein de la population active $^{8}$.

En outre, l'« indicateur de la participation des femmes » (IPF) est composé de variables définies explicitement pour mesurer le contrôle que les hommes et les femmes peuvent exercer sur leur destinée dans les domaines politique et économique. Ainsi, l’on rejoint la notion de «capabilité » proposée par 
Armatya Sen (1985). L'IPF prend en compte plusieurs critères, dont celui qui est relatif à la part des femmes dans le PIB par habitant. Comme autre critère, il y a la participation des femmes à la vie politique et à la prise de décision ; il est identifié au travers du pourcentage d'hommes et de femmes exerçant les fonctions de parlementaire. On a aussi comme critère ${ }^{9}$ le pourcentage d'hommes et de femmes qui exercent, d'une part les fonctions de direction et d'encadrement supérieur et, d'autre part, les professions techniques, libérales, etc.

Cette évolution idéologique a permis de mettre en exergue le rôle de premier plan joué ces dernières décennies par les femmes dans le système productif (produits éthiques), particulièrement en ce qui concerne les échanges commerciaux en tant qu'initiatives entrepreneuriales (sociales et solidaires) tant dans les pays du Nord que dans ceux du Sud. Peu importe qu'il se situe au niveau productif, managérial, de la gestion, de la création de ces unités de travail ou corresponde à une simple contribution (formelle ou informelle) à la croissance du système productif (Tchamanbe 1999).

Au Cameroun, cette implication, très remarquée des femmes, se fait plus vigoureuse, même si les activités commerciales qu'elles initient et dirigent sont seulement génératrices de revenus ou se déroulent dans de petites unités de production de produits éthiques. Cette dynamique s'exprime par la présence des femmes dans presque tous les secteurs (primaire, secondaire et tertiaire) d'activités économiques ou non économiques, et se déroule dans un contexte de mondialisation ${ }^{10}$ des économies et d'émergence des entreprises sociales et solidaires respectant des principes du commerce équitable.

\section{Analyse des fondements et des principes du commerce équitable pour une égalité de genre dans le commerce}

Développé depuis plusieurs années par différentes associations dans le monde, le commerce équitable obéit à des principes élémentaires simples permettant de garantir aux petits producteurs du Sud des conditions de vie décentes, et la possibilité de prendre en charge eux-mêmes leur développement. Il existe un grand nombre de produits échangés selon le modèle du commerce équitable. On distingue, cependant, deux grands groupes de produits équitables : les produits artisanaux (objets décoratifs, vêtements, bijoux, petits meubles, etc.) et les produits alimentaires de consommation courante, tels le sucre, les bananes, le cacao et le café. Ces deux catégories de produits appartiennent à des marchés fort différents, mais suivent les mêmes principes de base du commerce équitable, par exemple offrir un revenu plus élevé aux acteurs du Sud et réduire le nombre d'intermédiaires commerciaux. 
Au Cameroun, le commerce équitable concerne essentiellement les produits alimentaires (café, cacao, bananes, etc.) et artisanaux (sculptures sur bois, poterie, etc.) transformés et fabriqués localement par les femmes ou dans d'autres pays du Sud. Ses principes peuvent s'appliquer aux produits industriels. Le commerce équitable est une solution résolument moderne et efficace pour lutter contre les déséquilibres du commerce mondial et leurs conséquences dans les familles productrices de matières premières agricoles, forestières, etc. Parmi les dossiers à l'ordre du jour de la cinquième conférence ministérielle de l'Organisation mondiale du Commerce (OMC) de Cancun au Mexique de septembre 2003, figurait le commerce équitable.

Le commerce équitable, en établissant des relations de partenariat entre les parties prenantes (stakeholders), contribue au renforcement des échanges éthiques, économiques, sociaux, culturels, etc. pour un développement durable. Cette façon de commercer repose sur un certain nombre de principes.

Il existe une autre manière d'échanger, de faire du commerce, une autre manière de produire et de consommer. Depuis quelques années, le commerce équitable s'impose comme étant une alternative efficace pour réduire les inégalités entre le prix à la production et le prix à la consommation et redonner à la femme sa place dans les échanges commerciaux internationaux. Visant à rétablir un rapport d'échanges satisfaisants pour tous (du producteur au consommateur) basé sur l'égalité de genre, le commerce équitable est fondé sur les principes ci-après :

- Assurer une juste rémunération du travail des producteurs et artisans les plus défavorisés, leur permettant de satisfaire leurs besoins élémentaires : en santé, en éducation, en logement, en protection sociale, etc. ;

- Garantir le respect des droits fondamentaux des personnes (refus de l'exploitation des enfants, de l'esclavage, etc.) ;

- Instaurer des relations durables entre les différents acteurs et partenaires économiques ;

- Favoriser la préservation de l'environnement et du système écologique ;

- Proposer aux consommateurs des produits de qualité, des produits « éthiques » respectant les normes de production et la « traçabilité ». Les « produits éthiques » doivent au préalable respecter les règles fondamentales de production, les règles du droit de travail instituées par l'Organisation 
internationale du Travail (OIT) : interdiction du travail des enfants, interdiction du travail forcé, respect des libertés syndicales, etc.

Alors quels principes pour quels objectifs du commerce équitable en vue de l'égalité de genre ?

Pour participer au commerce équitable un certain nombre de principes doivent être respectés. Les produits éthiques sont achetés directement aux petits producteurs, généralement regroupés en coopératives, en groupes d'initiative commune, etc. c'est-à-dire exerçant dans le champ de l'entrepreneuriat social et solidaire. Le prix d'achat des produits au producteur est équitablement déterminé et constamment supérieur à celui du marché. Des systèmes de pré - financement de récoltes sont mis en place, ce qui permet d'éviter l'endettement coûteux des producteurs auprès des " usuriers ». Le partenariat d'échange, en d'autres termes, les relations contractuelles sont durables et basées sur les contrats de partenariat à part entière et à long terme. En contrepartie, les petits producteurs s'engagent à fournir des produits de qualité et respectant des normes. Les bénéfices sont affectés par l'entreprise sociale et solidaire elle-même suivant des principes démocratiques et servent la plupart du temps à financer le développement local et durable.

Le commerce équitable vise des objectifs très spécifiques. Il s'agit d'améliorer le sort des petits producteurs des pays du Sud, marginalisés par le manque de moyens matériels, financiers et d'expériences dans la production, la distribution, la commercialisation, etc. en créant des débouchés pour commercialiser leurs produits agricoles et artisanaux auprès des consommateurs des pays du Nord. L'objectif est aussi de constituer un réseau de consommateurs en sensibilisant l'opinion publique sur les injustices des règles du commerce international et en entreprenant des actions auprès des décideurs politiques et économiques afin de participer à une meilleure solidarité Nord-Sud pour un développement durable et équitable.

Créée en 1997, la plate-forme française pour le commerce équitable est le seul organisme national de représentation des acteurs du commerce équitable. Cette plate forme regroupe les importateurs (Solidar'Monde, Artisans du Monde, etc.), les importateurs-détaillants (Azimut - Artisans Monde, Alter Eco, Boutic Ethic, etc.), les boutiques (Artisans du soleil, Artisans du Monde, etc.), les associations de promotion (Association de solidarité aux Peuples d'Amérique, Échange dans l'organisation et la promotion des petits entrepreneurs (Echoppe), les associations de labellisation (Max Havelaar ${ }^{11}$ ), les structures de solidarité et d'échange dans l'organisation et la promotion des petits entrepreneurs (Comité catholique de lutte contre la faim, ingénieurs sans frontières, etc.). 
Au Cameroun, le commerce équitable est une réalité. En effet, dans le sous-secteur du bois, des artisans camerounais regroupés en association (Bois décor industrie, Harmony, Promoteur des masques, Noire Afrique promotion, Nodi bois, Association KKED, etc.) jouent un rôle important dans la transformation du bois. L'approche éthique de ces associations comporte trois stades : l'approvisionnement, la transformation et la distribution. Les différents bois (bubinga, bété, iroko, sapelli, etc.) utilisés dans les ateliers des artisans sont issus essentiellement des forêts communautaires camerounaises (regroupées en coopératives, en groupes d'initiative commune, etc.). Les autres intrants sont achetés dans le circuit commercial formel respectant une certaine éthique. La transformation de ce bois respecte les règles de sécurité et de législation internationale du travail notamment l'interdiction du travail d'enfants mineurs, du travail forcé et d'égalité de genre dans la rémunération.

Le circuit de distribution permet d'établir le « juste prix » (Fair Price) du produit fabriqué qui tient compte des coûts réels de production (et non de subir le prix des multinationales et des grandes chaînes de distribution). Les distributeurs évitent de tomber sous le coup de spéculations commerciales. Les producteurs doivent recevoir un prix « équitable » pour la vente de leurs produits, c'est-à-dire un prix qui permet aux producteurs et artisans du Sud de recevoir un revenu vital minimum. Les organisations de commerce équitable du Nord (OCE) doivent donc offrir un revenu de subsistance (living wage) qui permette aux artisans ou aux petits producteurs de satisfaire leurs besoins de base ainsi que ceux de leurs familles, tels que les besoins d'alimentation, de logement, d'éducation et de santé (Tadros et Malo 2002).

En matière de partenariat ${ }^{12}$ pour une égalité de sexe et pour un développement durable, des relations commerciales sont établies entre les propriétaires des forêts communautaires camerounaises réunis pour la production du bois (dont le but est de préserver la nature, les ressources, les essences rares ; d'éviter le pillage des essences et d'encourager la transformation locale du bois), les associations des artisans (sculpteurs, menuisiers, etc.) pour la transformation et les associations ou les boutiques situées en Occident exerçant dans le commerce équitable pour la distribution. Dans un partenariat inscrit sur le commerce équitable, deux entreprises sociales et solidaires françaises commercialisent sur le marché européen des produits fabriqués par Bois décor industrie Cameroun. En effet, en septembre 2000 Bois décor industrie a signé un contrat de partenariat avec Ethnic. Org pour la fabrication des porte-documents, des plumiers et dessous-de-plats en bois lamellés collés et en 2002 avec Suds-sarl (label EDEA) pour la fabrication du mobilier de maison et d'art (tables, chaises, étagères, consoles, etc.). 


\section{Conclusion}

Dans une approche sociale et solidaire du commerce équitable, il s'agit de travailler d'abord avec les producteurs les plus défavorisés des pays du Sud, dans le cadre d'un développement durable. Aussi il faut refuser systématiquement une quelconque forme d'esclavage ou de travail forcé, y compris l'exploitation des enfants, surtout des mineurs et aussi d'inégalité de genre. Il s'agit ensuite de signer des contrats entre les différents partenaires (particulièrement en intégrant les femmes) en présence et d'établir des garanties qui portent principalement sur : le prix du produit qui permet une juste et équitable rémunération (Fair Remuneration) des différents intervenants économiques. Cette rémunération prend en compte leurs besoins et ceux de leurs familles, notamment en termes de formation, de santé, de protection sociale, etc. ; la qualité et la traçabilité des produits ; le versement d'un acompte surtout, lorsque les organisations de producteurs n'ont pas le fonds de roulement nécessaire pour acheter la matière première, pour survivre tout simplement entre la commande et le règlement final, la période de soudure ou les délais de livraison.

Cette approche entrepreneuriale doit également privilégier des relations commerciales et d'échange durables avec les producteurs pour un développement durable. Pour eux, c'est la durée qui assure l'avenir. Assurer la transparence dans le fonctionnement des différents partenaires, qui passe par une information réciproque à chaque étape sur les conditions de travail, les salaires, la durée des relations, les processus de production et de distribution, les prix, les marges, etc. Accepter le contrôle sur le respect de ces principes, à chaque étape du processus.

En plus, il existe des moyens pour intégrer les différentes parties prenantes (stakeholders), particulièrement les femmes, dans le commerce mondial et dans les processus formels de prise de décision, bien que le cadre légal puisse être limitant (Tadros et Malo 2002). Un tel désir d'inclusion et d'égalité de genre requiert donc une certaine transformation du modèle entrepreneurial qui peut être associatif ou coopératif. Or l'entrepreneuriat social et solidaire des femmes, en produisant des produits éthiques, fait preuve d'originalité surtout dans le sens du commerce équitable pour une participation équitable à l'échange international. Il permet de mobiliser d'autres ressources et produits que ceux rencontrés habituellement dans une entreprise marchande. Des contrats de partenariat entre les entreprises du Nord et du Sud et des statuts mieux élaborés et adaptés aux spécificités des entreprises sociales et solidaires du commerce équitable pourraient permettre de relever les défis de cette nouvelle forme d'entrepreneuriat et de cette nouvelle façon de commercer, d'échanger qui intégrerait l'approche genre. 
Le commerce équitable a des limites. Ce n'est pas de la « charité », se défend une productrice malienne de coton labellisé Max Havelaar. " Nous travaillons dur et élaborons un produit de très bonne qualité pour lequel nous recevons une rémunération juste ». Le "prix juste » est-il défini par le producteur ou le distributeur? Dans les chiffres cités plus haut, peut-on remarquer que les millions d'euros issus du commerce équitable ne retournent pas dans les pays du Sud producteurs ? Une contrainte est aussi le fait que les producteurs qui souhaitent être labellisés n'ont vraiment pas de marge de manœuvre d'autant plus qu'il y a déjà un cahier de charges et des règles de production à respecter.

De plus, la majorité des produits éthiques du commerce équitable sont destinés à l'exportation. On retombe donc dans un " cercle vicieux » dans lequel les productions du Sud sont fonction de la demande du Nord. Le commerce équitable envisage la transformation de certains produits sur place et d'autres ailleurs. Il crée certes plus d'emplois dans les pays du Sud, mais beaucoup plus d'emplois fortement rémunérés et plus de richesses dans les pays du Nord qui refusent de baisser leurs différentes taxes.

\section{Notes}

1. L'entrepreneuriat social et solidaire n'est pas un concept stable. C'est une notion qui se modifie, qui évolue et qui se complexifie dans le temps et dans l'espace. En effet, elle se positionne en réponse aux mutations des systèmes d'échange, des systèmes économiques, sociaux, écologiques et techniques dominants. L'économie sociale et solidaire regroupe au Cameroun les coopératives, les groupes d'initiative commune, les groupements d'intérêt économique, les mutuelles, les organisations paysannes, les organisations non gouvernementales et les associations qui produisent des biens et services. Ses différentes composantes adhèrent à certains principes fondateurs et respectent des règles de fonctionnement qui fondent leur unité et leur spécificité : l'épanouissement de la personne humaine et sa primauté sur le capital, le développement de la production et de l'échange au service de la satisfaction des besoins et non de la valorisation des capitaux, le caractère démocratique de la gestion, l'indépendance à l'égard des pouvoirs publics, la libre adhésion des personnes.

2. Ces petits producteurs sont totalement démunis face à des multinationales qui imposent leur prix et leurs conditions de travail. Ils sont incapables d'élaborer des stratégies de développement à long terme (diversification, investissement, amélioration des techniques de production, etc.). Le développement est bloqué, la situation précaire et les écarts se creusent.

3. Le développement durable apparaît comme une nouvelle forme de développement de l'humanité qui respecte l'environnement global et les équilibres écologiques fondamentaux qui régissent tant les milieux continentaux 
qu’océaniques. L’objectif final est de répondre aux besoins actuels sans compromettre l'aptitude des générations futures à répondre aux leurs.

4. Nous nous sommes largement inspirés dans cette partie des travaux de Mark Lansky (2000 et 2001).

5. Pour la synthèse des références bibliographiques sur cette question, voir Korabik (1999).

6. Le récent débat sur le travail de nuit des femmes montre que la question de John Stuart Mill sur la justice reste d'une frappante actualité. D’après lui, depuis plus de 130 ans, on a posé la question suivante : la justice peut-elle tolérer que l' « on refuse aux femmes la part d'honneur et de distinction qui leur revient, et qu'on leur refuse le droit qui échoit à tous les êtres humains de choisir leur profession [...] selon leur inclinaison et à leurs propres risques ».

7. Pour une analyse et un examen détaillés se référer aux contributions de Paul Collier, Ingrid Palmer et d'autres auteurs sur ce sujet. Voir aussi les travaux de Razavi et Miller (1995) et Razavi (1997).

8. En 1999, l’IDSH a été calculé par le PNUD pour 103 pays. Ainsi pour évaluer les inégalités entre sexes, deux comparaisons sont généralement distinguées : la valeur de l'IDSH d'un pays comparée avec celle de son IDH et le classement de ce pays selon l'IDSH par rapport à son classement selon l'IDH. Autrement dit, lorsque l'IDSH est proche de l'IDH, il y a moins d'inégalité entre les hommes et les femmes. Dans tous les pays, l'IDSH est inférieur en valeur à l'IDH, ce qui veut dire que, pour l'échantillon de pays en question, il y a des inégalités entre les hommes et les femmes. Lorsque le classement de l'IDSH est inférieur au rang obtenu pour l'IDH, cela signifie que les avancées moyennes dans le développement humain ne sont pas réparties équitablement entre les hommes et les femmes. Lorsque se produit le contraire, on a un développement humain réparti de façon plus équitable entre les deux sexes.

9. Pour de plus amples explications et précisions, voir PNUD (1999).

10. La mondialisation est un état du monde dominé par une libéralisation poussée des économies et des firmes dont la logique réside dans la réalisation de leurs objectifs, leurs intérêts et leur pérennité.

11. Max Havelaar est l'un des principaux labels européens, très implanté aux PaysBas, en Suisse et en France notamment. Cette structure agit pour développer le commerce équitable dans près de 20 pays producteurs et garantit 6 catégories de produits : principalement le café mais aussi le miel, la banane, le cacao, le thé et le sucre.

12. Le partenariat s’établit aussi dans le domaine de la formation. En effet, les artisans du Sud reçoivent de leurs partenaires du Nord des formations dans le but de respecter les normes de production et d'améliorer la qualité et la traçabilité des produits.

\section{Références}

Albert, O., 1998, « Le double objectif du commerce équitable » et « Des partenairesproducteurs au Sud », Pour un commerce équitable : expériences et propositions 
pour un renouvellement des pratiques commerciales entre les pays du Nord et ceux du Sud, Paris : Éditions Charles Léopold Mayer : 15-18 et 45-61.

BIT, 2000, Genre! Partenaires et égaux,Genève, Bureaude l’Égalité entreHommes et Femmes.

Blackden, C. M. et Bhanu, C., 1999, Inégalité des sexes, croissance et réduction de la pauvreté, Programme spécial d'assistance pour l'Afrique, Rapport sur la pauvreté en Afrique subsaharienne de 1998, Washington : Banque mondiale.

Boncler, J., 2002, « L'économie solidaire : une nouvelle forme d'entrepreneuriat ? », Actes du 2e Congrès de l'Académie de l'Entrepreneuriat, des 17 et 18 avril 2002, Bordeaux : 77-95.

Boserup, E., 1983, La femme face au développement économique, Paris : PUF.

Brown, M. B. etA dam, S., 1999, « Le commerce équitable dans les échanges NordSud », J. Defourny, Develterre P. et B. Fonteneau (sous la direction de), L'économie sociale au Nord et au Sud, Belgique : De Boeck Université : 278 p. Caubergs, L., 1997, « Les femmes entrepreneurs et les ONG d’appui en Afrique subsaharienne : un éloge de la diversité et de la complexité », Rapport final : Recheche-Action sur l'entrepreneuriat féminin en Afrique subsaharienne, Leuven, ATOL : 125 p.

Korabik, K., 1999, « Sex and gender in the new millennium », G. N. Powell (ed.), Handbook of gender and work, Powell G. Thousand Oaks, Sage: 3-16.

Lansky, M., 2000, « Du genre, des femmes et de tout le reste », Partie I, Revue internationale du Travail, vol. 139, $\mathrm{n}^{\circ} 4$ : 539-566.

Lansky, M., 2001, « Du genre, des femmes et de tout le reste », Partie II, Revue internationale du Travail, vol. 140, $\mathrm{n}^{\circ} 1$ : 95-131.

Lesdain, S. B., 1999, Femmes camerounaises en région parisienne, Paris : L'Harmattan.

PNUD, 1999, Rapport mondial sur le développement humain, Paris : Éditions de Boeck et Larcier.

Polanyi, K., 1985, La grande transformation, Paris : Gallimard.

Prades, J., 1985, La création - destructrice, Paris : L’Harmattan.

Razavi, S. et Miller, C., 1995, « From WID to GAD: conceptual shifts in the women and development discourse » [Document de travail UNSRID n ${ }^{\circ} 1$, Quatrième Conférence mondiale des Nations Unies sur les Femmes, février, Genève].

Razavi, S., 1997, « Fitting gender into development institutions », World Development, Oxford, vol. 25, n7, juillet: 1111-1125.

Rispal, H., 2002, « Entreprendre en économie solidaire », Actes du 2e Congrès de l'Académie de l'Entrepreneuriat, des 17 et 18 avril 2002, op. cit. : 277-289.

Sen, A., 1985, Commodities and capabilities, Amsterdam/New-York: Oxford University Press.

Sen, A., 2000, Repenser l'inégalité (Inequality reexamined, 1992), Paris : Le Seuil. Sen, A., 2000, Un nouveau modèle économique: Développement, justice, liberté, Paris : Éditions Odile Jacob. 
Tadros, C. et Malo, M. C., 2002, « Commerce équitable, démocratie et solidarité : Equal Exchange, une coopérative exceptionnelle au Nord », Revue Nouvelles pratiques sociales, Vol. 15, numéro $1: 17$ p.

Tchamanbe, D. L., 1999, « La femme camerounaise face aux enjeux économiques du XXIe siècle » [Communication au Colloque sur « Femmes, leadership et développement : un regard prospectif sur le 21ème siècle », organisé par le MINCOF, Yaoundé, mai].

Tchamanbe, D. L. et Tchouassi, G., 2003, «Femmes camerounaises : renforcement de leurs capacités entrepreneuriales par la formation », B. Ponson et M. Niculescu, (sous la direction de), «La formation à l'entrepreneuriat », Actes des VIIes Journées scientifiques du Réseau Entrepreneuriat de l'AUF, Ile Maurice, 4-6 juillet 2001 : 167-176.

Tchouassi, G., 2000, « Femmes entrepreneurs au Cameroun : une approche par les récits de vie », Revue congolaise de Gestion, Numéro double 02-03, janvierdécembre : 63-77.

Tchouassi, G., 2000, « Les comportements d'épargne des femmes au Cameroun : une analyse à partir de leurs récits de vie», African Review of Money, Finance and Banking, Supplementary issue of Saving and Development : 117-133.

Tchouassi, G., 2002, «Entreprendre au féminin au Cameroun : possibilités et limites », Actes du 2e Congrès de l'Académie de l'Entrepreneuriat, des 17 et 18 avril, op. cit. : 509-521.

Tchouassi, G., 2002, « Épargne des femmes au Cameroun : épargne individuelle, épargne collective ou épargne solidaire » [Communication aux deuxièmes rencontres inter-universitaires sur le thème "Sens et portée de l'économiesolidaire ", Lyon, 5-7 février].

Touna Mama, 1998, (sous la direction de), La Mondialisation et l'économie camerounaise, Yaoundé : Édition Saagraph-Friedrich-Ebert-Stiftung.

Sites Internet consultés :

- www.andines.com

- www.artisans-du-soleil.com

- www.artisansdumonde.org

- www.bouticethic.com

- www.EDEA.fr

- www.equalexchange.com

- www.penserpouragir.org

- www.solidarmonde.fr 